\title{
The origin of noise and magnetic hysteresis in crystalline permalloy ring-core fluxgate sensors
}

\author{
B. B. $\operatorname{Narod}^{1,2}$ \\ ${ }^{1}$ Narod Geophysics Ltd., Vancouver, Canada \\ ${ }^{2}$ Department of Earth, Ocean and Atmospheric Sciences, University of British Columbia, Vancouver, Canada
}

Correspondence to: B. B. Narod (bnarod@eos.ubc.ca)

Received: 19 March 2014 - Published in Geosci. Instrum. Method. Data Syst. Discuss.: 19 June 2014

Revised: 17 August 2014 - Accepted: 26 August 2014 - Published: 29 September 2014

\begin{abstract}
Developed in the 1960s for use in highperformance ring-core fluxgate sensors, 6-81.3 Mo permalloy remains the state of the art for permalloy-cored fluxgate magnetometers. The magnetic properties of 6-81.3, namely magnetocrystalline and magnetoelastic anisotropies and saturation induction, are all optimum in the $\mathrm{Fe}-\mathrm{Ni}-\mathrm{Mo}$ system.

In such polycrystalline permalloy fluxgate sensors, a single phenomenon may cause both fluxgate noise and magnetic hysteresis; explain Barkhausen jumps, remanence and coercivity; and avoid domain denucleation. This phenomenon, domain wall reconnection, is presented as part of a theoretical model. In the unmagnetized state a coarse-grain highquality permalloy foil ideally forms stripe domains, which present at the free surface as parallel, uniformly spaced domain walls that cross the entire thickness of the foil. Leakage flux "in" and "out" of alternating domains is a requirement of the random orientation, grain by grain, of magnetic easy axes' angles with respect to the foil free surface. Its magnetostatic energy together with domain wall energy determines an energy budget to be minimized. Throughout the magnetization cycle the free-surface domain pattern remains essentially unchanged, due to the magnetostatic energy cost such a change would elicit. Thus domain walls are "pinned" to free surfaces.

Driven to saturation, domain walls first bulge then reconnect via Barkhausen jumps to form a new domain configuration that I have called "channel domains", which are attached to free surfaces. The approach to saturation now continues as reversible channel domain compression. Driving the permalloy deeper into saturation compresses the channel domains to arbitrarily small thickness, but will not cause them to denucleate. Returning from saturation the channel domain
\end{abstract}

structure will survive through zero $H$, thus explaining remanence. The Barkhausen jumps, being irreversible exothermic events, are sources of fluxgate noise powered by the energy available from domain wall reconnection.

A simplified domain energy model can then provide a predictive relation between ring-core magnetic properties and fluxgate sensor noise power. Four properties are predicted to affect noise power, two of which are well known: saturation total magnetic flux density and magnetic anisotropy. The two additional properties are easy axes alignment and foil thickness. Flux density and magnetic anisotropy are primary magnetic properties determined by an alloy's chemistry and crystalline lattice properties. Easy axes alignment and foil thickness are secondary, geometrical properties related to an alloy's polycrystalline fabric and manufacture. Improvements to fluxgate noise performance can in principle be achieved by optimizing any of these four properties in such a way as to minimize magnetostatic energy.

Fluxgate signal power is proportional to $B-H$ loop curvature $\left[\mathrm{d}^{2} B / \mathrm{d} H^{2}\right]$. The degree to which Barkhausen jumps coincide with loop curvature is a measure of noise that accompanies the fluxgate signal. $B-H$ loops with significant curvature beyond the open hysteresis loop may be used to advantage to acquire the fluxgate signal with reduced noise.

\section{Introduction}

The 1979 launch of the NASA spacecraft MAGSAT marked a milestone in the development of magnetometry. In particular, it terminated an effort by a group at the Naval Ordnance Laboratory, led by Daniel Israel Gordon, to create 
a low-noise magnetic alloy now referred to as 6-81.3 Mo permalloy, or simply "6-81" (http://www.magson.de/ technology/tech3.html) (Gordon et al., 1968). Answering the question of why 6-81.3 Mo permalloy became the de facto standard for low-noise ring-core fluxgates is the first motivation for this paper.

I begin with an examination of data that relate fluxgate noise to various physical parameters and provide evidence that the geometric configurations of a fluxgate's magnetic material are significantly more important than Gordon's investigators may have thought. As surface area - either free surface or intergrain - of the magnetic material increases, so does the fluxgate noise power. This suggests there is a causal link between fluxgate noise and magnetostatic energy by way of domain wall energy, and that all are fundamentally dependent on the properties magnetic moment and anisotropy.

Amos et al. (2008) using magnetic force microscopy (MFM) have shown that sputtered permalloy films assume configurations called "stripe domains". Thus it should be beneficial to examine the causes of stripe domains. Since fluxgate sensor function requires periodic core saturation in order to generate a signal, it is also beneficial to study the evolution of stripe domains throughout the magnetization cycle.

Recently, Coïsson et al. (2009), also using MFM, determined the surface expression of stripe domains in sputtered films throughout the magnetization cycle, confirming constraints on magnetostatic energy which can be derived from simple principles. I propose that, on approach to saturation, each stripe domain undergoes a well-defined, exothermic and irreversible transition to a saturated state, with domain wall loss supplying the energy. It further follows that these transitions could be used to explain many aspects of irreversible and reversible magnetization processes in magnetically soft materials, in particular DC coercivity, remanence and hysteresis.

Fluxgate noise and DC hysteresis are thus arguably two facets of the same phenomenon, and fluxgate sensor noise is causally tied to domain wall energy by a simple relationship: fluxgate noise is proportional to available domain wall energy, which itself is a function of domain size and domain wall energy density.

Magnetocrystalline anisotropy complicates the story: in any polycrystalline permalloy, each crystal assumes an easy axis from a distribution, an axis that in general will not lie parallel to the magnetizing field, nor to the free surface. This affects domain size and local magnetization processes. Saturation transitions in one crystal will not in general coincide with those in any other crystal. Fluxgate noise and a ferromagnet's path to saturation will be an aggregate of all the individual crystals' processes. But the basic phenomena can be understood by examining processes in a single, ideal crystal.

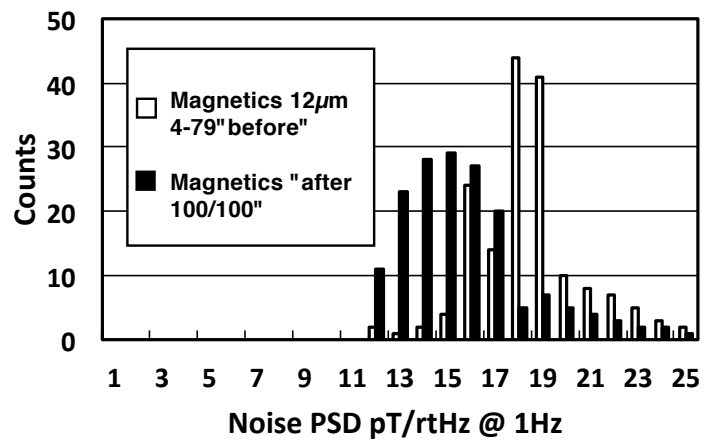

Figure 1. Histograms of noise power for 4-79 permalloy ring cores.

\section{Magnetic properties of 6-81}

The original choice of 6-81 followed on the work of Pfeifer (1966) and Pfeifer and Boll (1969), who presented data which showed that 6-81 has near-zero polycrystalline magnetostriction and magnetocrystalline anisotropy for very slow cooling. The higher molybdenum content of 6-81 has additional useful aspects, the first of these being a lower saturation total magnetic flux density $B_{\mathrm{s}}$, now known to directly affect the noise power level of a magnetometer. The first indications that lower $B_{\mathrm{S}}$ led to lower fluxgate noise were provided by Shirae (1984) and Narod et al. (1985), both using selections of amorphous alloys.

Another useful aspect to using 6-81 is its need for very slow cooling rates to achieve low anisotropy. While the added furnace operating time is a significant processing cost, it results in lower average noise power levels, as compared with those for a rapidly cooled alloy such as 4-79 permalloy. Figure 1 presents the results from this author's own study of 167 Magnetics Inc. 4-79 ring cores with properties otherwise similar to Infinetics S-1000 6-81 ring cores. These rings were made from 4-79 square loop permalloy, with seven layers each $12 \mu \mathrm{m}$ thick. The white histogram shows the noise power probability density function (PDF) for the rings as received from their original heat treatment. The black histogram shows the noise power PDF for the same rings after they had all received an extra heat treatment of $100 \mathrm{~h}$ at $100^{\circ} \mathrm{C}$ in air. Note the general shift of the density function towards lower noise levels. Figure 2 shows a similar probability density function for a collection of 195 as-received 6-81 ring cores $(12 \mu \mathrm{m})$, a function which also exhibits an asymmetric form. These data suggest that $6-81$ 's requirement for slow cooling acts as a substitute for the beneficial low-temperature heat treatment.

The choice of 6-81 for fluxgate sensors thus has four distinct advantages. It simultaneously minimizes magnetocrystalline anisotropy, magnetostriction and $B_{\mathrm{S}}$ and, by slow cooling, also achieves a preferred statistical distribution for average noise power. It is clearly the optimum composition 


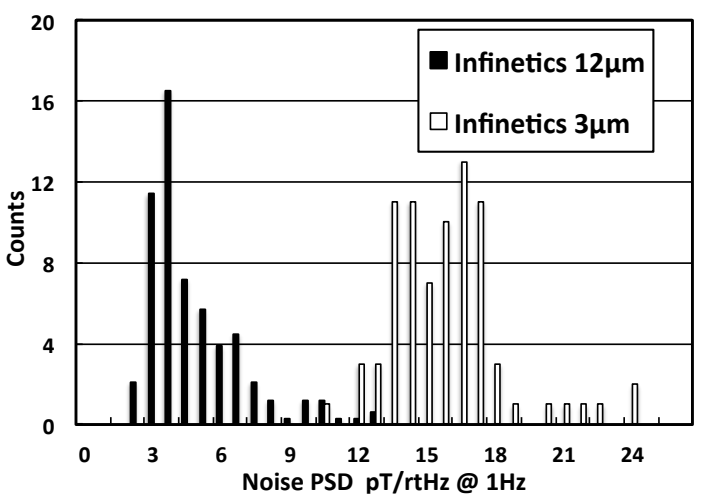

Figure 2. Histograms of noise power for 6-81 permalloy ring cores.

in the $\mathrm{Fe}-\mathrm{Ni}-\mathrm{Mo}$ ternary system. These properties can now provide clues to the nature of fluxgate sensor noise.

\section{Geometrical evidence towards a theory of noise}

Two more observations provide evidence for the last clues needed for a theory of noise. Figure 3 shows $\mathrm{d} B / \mathrm{d} t$ vs. $t$ plots, an analog for DC differential permeability vs. $H$ for five ring cores, two of material 6-81 and three of 479 permalloy, each annotated with its noise power. Lower noise power correlates with higher peak $\mathrm{d} B / \mathrm{d} t$. Similarly amorphous alloys treated at temperatures up to $350{ }^{\circ} \mathrm{C}$ will develop large magnetic domains (Shishido et al., 1985), and treating such alloys to about $300^{\circ} \mathrm{C}$ will significantly reduce their noise power (Narod et al., 1985). The 4-79 rings exhibit both higher noise power and higher coercivity (peaks are to the right of the 6-81 curves). This is likely a grain size effect (Pfeifer and Radaloff, 1980).

A hypothesis follows: (1) larger domain sizes lead to both lower noise and to higher differential permeability, and (2) smaller grain size leads to higher domain wall energy, noise power and coercivity. It then follows that total domain wall area is also related to fluxgate sensor noise.

A last clue comes from my examination of noise in a large collection of Infinetics 6-81 ring cores. This included 195 $12 \mu \mathrm{m}$ rings with 7 layers each, and $753 \mu \mathrm{m}$ rings with 28 layers. Otherwise these rings are similar. Figure 2 shows the noise PDFs for this collection, with $3 \mu \mathrm{m}$ rings' data in white, and $12 \mu \mathrm{m}$ data in black. Counts have been scaled to match. These data clearly show that the $3 \mu \mathrm{m}$ rings are typically $12 \mathrm{~dB}$ noisier than the $12 \mu \mathrm{m}$ rings. Equally, the $3 \mu \mathrm{m}$ rings have 4 times the free-surface area, which is the same ratio. With such a large number of specimens, from a large number of independent processing batches, this statistic is robust and cannot have happened by chance. Clearly the geometry of the fluxgate core material matters, and any theory of fluxgate noise must be able to take this into account.

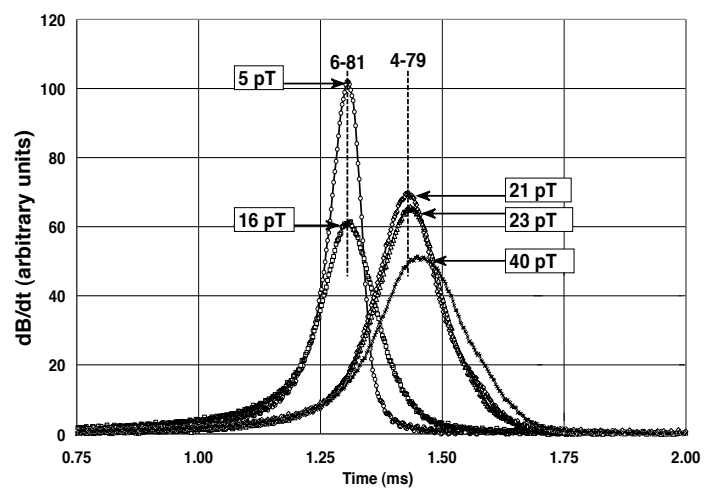

Figure 3. $\mathrm{d} B / \mathrm{d} t$ plots for $6-81$ and $4-79$ ring cores. Noise values are in $\mathrm{pTrtHz}^{-1}$ at $1 \mathrm{~Hz}$, for this insert and all other data in this paper.

\section{The $B-H$ curve from a fluxgate's point of view}

Consider a ring core lying in the plane of this page, immersed in a small external field $H_{\mathrm{e}}$ (Fig. 4), with core drive field $H_{\mathrm{d}}$; internal flux density $B$. Consider a volume element $A-A^{\prime}$, comprising two mirror-symmetric elements $180^{\circ}$ apart on the ring and located where $H_{\mathrm{e}}$ is parallel to $H_{\mathrm{d}}$. Locally at point $A, B$ is $B\left(H_{\mathrm{d}}+H_{\mathrm{e}}\right)$ and at $A^{\prime} B$ is $B\left(H_{\mathrm{d}}-H_{\mathrm{e}}\right)$. As viewed from a sensor winding parallel to $H_{\mathrm{e}}$, their combined flux density is

$\Delta B\left(H_{\mathrm{d}}\right)=B\left(H_{\mathrm{d}}+H_{\mathrm{e}}\right)-B\left(H_{\mathrm{d}}-H_{\mathrm{e}}\right)=H_{\mathrm{e}} \frac{\mathrm{d} B}{\mathrm{~d} H_{\mathrm{d}}}$.

This requires that one superpose the ring's response to $H_{\mathrm{d}}$ and $H_{\mathrm{e}}$ (Primdahl et al., 2002). $H_{\mathrm{e}}$ is not necessarily uniform. It can vary both spatially and temporally. Open-circuit sensor windings avoid temporal changes, while short-circuit or capacitively loaded sensors cause $H_{\mathrm{e}}$ to vary throughout the saturation cycle.

Moving up the $B-H$ curve by an amount $\partial H_{\mathrm{d}}$, the change in flux density as seen by the sensor winding is

$\Delta B\left(H_{\mathrm{d}}+\partial H_{\mathrm{d}}\right)-\Delta B\left(H_{\mathrm{d}}\right)=\partial H_{\mathrm{d}} H_{\mathrm{e}} \frac{\mathrm{d}^{2} B}{\mathrm{~d} H_{\mathrm{d}}^{2}}$.

The rate of change of flux density with time is then

$\frac{\mathrm{d} B}{\mathrm{~d} t}=H_{\mathrm{e}} \frac{\mathrm{d}^{2} B}{\mathrm{~d} H_{\mathrm{d}}^{2}} \frac{\mathrm{d} H_{\mathrm{d}}}{\mathrm{d} t}$.

Similarly, for a general volume element $C-C^{\prime}$, one takes into account $B-H$ misalignment. To calculate energy available to the sensor winding per cycle, one needs to multiply $\mathrm{d} B / \mathrm{d} t$ by $H_{\mathrm{e}}$, and integrate over volume and time:

energy $=\int_{T} \int_{V}\left(\frac{\mathrm{d}^{2} B}{\mathrm{~d} H_{\mathrm{d}}^{2}} \cdot H_{\mathrm{e}}\right)\left(\frac{\mathrm{d} H_{\mathrm{d}}}{\mathrm{d} t} \cdot H_{\mathrm{e}}\right) \mathrm{d} V \mathrm{~d} t$. 
Thus fluxgate sensitivity results from the second derivative of the $B-H$ curve, i.e., its curvature. Actual power transfer goes as the product of $B-H$ curvature and the rate of change of the drive field. The importance of the second derivative was originally noted by Marshall (1966) and Primdahl (1970).

\section{A theory of noise}

To understand how saturation can cause both signal and noise, it is necessary to examine the processes that govern the formation and evolution of the ring's magnetic domains. For an idealized iron crystal, Fig. 5a-d respectively represent a demagnetized ring core, a core immersed in an external field, a core partially magnetized by an excitation field and a core saturated by an excitation field. Each sector representation $(\mathrm{a}-\mathrm{c})$ comprises two domains jointly bounded by a common $180^{\circ}$ domain wall. In Fig. 5a the core is depicted as having equal opposed domain paths, with zero net magnetization.

In an external field $H$ (Fig. 5b), only sectors with magnetization directions parallel to $H$ respond. A sensing winding develops a self-inductance higher than for a saturated core, and can generate the fluxgate signal as described above. In Fig. 5c an excitation field has the domain path assisted by the excitation field expand, consistent with the high effective permeability one expects in a toroidal magnetic circuit. Here the effects of the excitation and external fields are entirely separable with the excitation field occupying a toroidal, low-reluctance circuit and the external field response occupying a solenoidal, high-reluctance circuit. Perfect separability also manifests as zero mutual inductance between the two magnetic circuits. At saturation (Fig. 5d), both ferromagnetic paths vanish, and inductances fall to air-core values (Butta and Ripka, 2008).

Figure 5 is not useful for considering domains in polycrystalline permalloys. I present here a hypothetical hysteresis and noise model based on stripe domains. Here domains have uniform width $D$, occupy the entire thickness $t$ of the film (or foil, or grain), and have lengths that are large compared to either width or thickness. Typically $D$ and $t$ are of the same magnitude, with $D<t$, except for very thin films. Figure 6 extends the concepts of Fig. 5 to examine a small portion of a core, within a single crystal - one that occupies the entire thickness of the core foil material.

Areal domain wall energy density can be approximated as

$E_{\mathrm{d}}=2 \pi \sqrt{\frac{J S^{2} K}{a}} \propto 2 \pi \sqrt{\frac{B_{\mathrm{s}}^{2} K}{a}}$.

$K$ is a parameter representing all of magnetic anisotropy, $J$ is the exchange integral, $S$ is the magnitude of atomic spin and $a$ is the lattice constant (Chikazumi, 1997). $B_{\mathrm{s}}^{2}$ is proportional to $J S^{2}$ given that $J$ is proportional to Curie temperature $T_{\mathrm{c}}$ (Weiss, 1948), $T_{\mathrm{c}}$ is proportional to magnetic moment-squared $M^{2}$ (Chikazumi, 1997, Eq. 6.8), and $B_{\mathrm{S}}$ correlates strongly with $M$ (see Sect. 7 below). The substitution with $B_{\mathrm{S}}$ requires the assumption that face-centered cubic lattice parameters, spin magnitude and molecular field coefficient vary little or not at all compared with variation in $B_{\mathrm{S}}$ over the range of permalloy compositions.

Magnetic anisotropy for cubic alloys has higher order terms. Reducing anisotropy to a single parameter, however, does not significantly impact our aim, which is to calculate total domain wall energy and total magnetostatic energy, and minimize their sum in order to determine domain width. Magnetostatic energy is required on the free surface of a permalloy foil as all crystalline permalloys have nonzero anisotropy and an easy axis that dips across the free surface at some angle $\alpha$. Flux density across the free surface is thus about $\alpha B_{\mathrm{s}}$, where a small angle is assumed for $\alpha$. Figure 6 indicates the associated flux paths as curved arrows originating from one domain and returning to an adjacent domain.

For soft magnetic materials the calculation is slightly more complicated, with three energies to equilibrate: domain wall energy $E_{\mathrm{d}}$, magnetostatic energy $E_{\mathrm{s}}$ and magnetic anisotropy energy $E_{\mathrm{c}}$. Assuming uniform spin rotation and neglecting free-surface depolarization, these energies (per unit volume) are

$$
\begin{aligned}
& E_{\mathrm{d}} \propto 2 \pi \frac{\sqrt{B_{\mathrm{s}}^{2} K / a}}{D} ; E_{\mathrm{s}}=\left(\beta B_{\mathrm{S}}\right)^{2} \frac{D}{t \mu_{\mathrm{o}}} \\
& E_{\mathrm{c}}=(\alpha-\beta)^{2} K,
\end{aligned}
$$

where $\beta$ is the actual magnetization dip angle with respect to the free surface. Similar to the approach taken in Butta and Ripka (2008), the total energy is minimized when $E_{\mathrm{d}}$ equals $E_{\mathrm{s}}$ and when the dip angle is

$\beta=\alpha \frac{1}{1+\frac{D}{t} \frac{B_{\mathrm{s}}^{2}}{K \mu_{\mathrm{o}}}}$.

For the thick foils used in typical ring cores the two angles become equal, and $D$ is proportional to $t^{1 / 2}$. In this case $E_{\mathrm{c}}$ vanishes and the equilibrium approaches that derived by Kittel (1949). In Amos et al. (2008), for films below $1 \mu \mathrm{m}$, a square-root relation between $D$ and $t$ held, implying a value for $K$ such that the angle $\beta=\alpha$.

The above calculation is for uniformly spaced, flat domain walls. When an external field is applied in a direction along the length of the domains, the initial response is to move the domain walls such that net magnetization adapts to the applied field. However the magnetostatic energy constraint at the free surface requires that the domains' surface expression remains essentially unchanged at a uniform distribution, i.e., $50 \%$ magnetic flux in and $50 \%$ out. (Any deviation from 50-50 would require a very large energy expense. Magnetostatic energy $E_{\mathrm{s}}$ could no longer be localized near the free surface.) It follows that, for a small field, the domain walls must adapt as shown in Fig. 7. $E_{\mathrm{s}}$ increases with negligible surface change, while domain wall energy increases due 

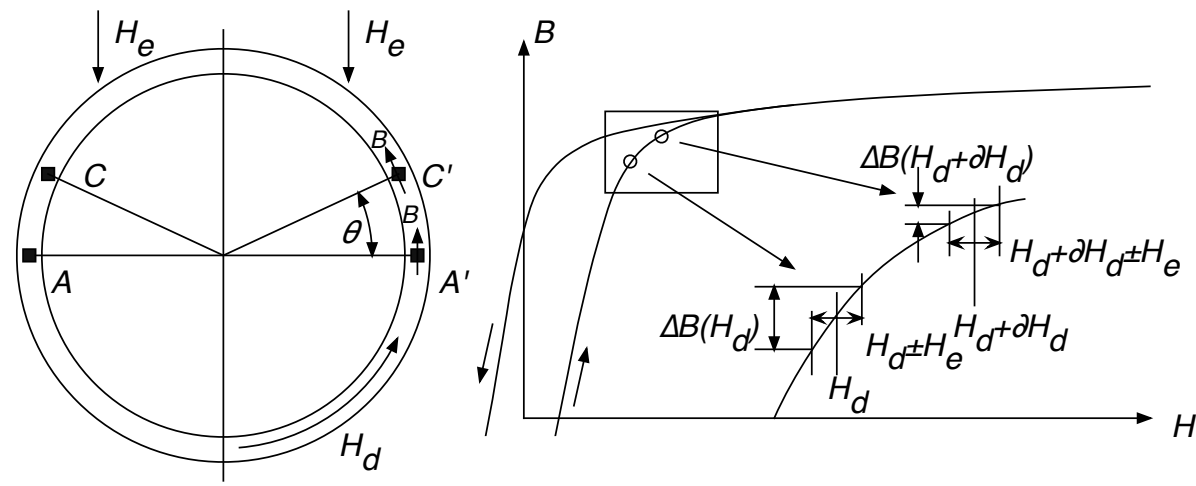

Figure 4. Ring core schematic and $B-H$ loop detail.
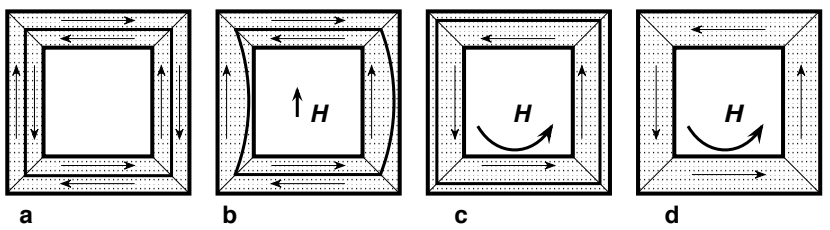

Figure 5. Ring-core domains: responses to external or excitation fields: (a) a demagnetized ring core; (b) a ring core exposed to external field $H$, depicting enhanced domains with magnetization parallel to $H$; (c) a ring core exposed to a drive field $H$, depicting enhanced domains with magnetization parallel to $H$; (d) a ring core exposed to a saturating drive field $H$, depicting single domains with magnetization parallel to $H$.

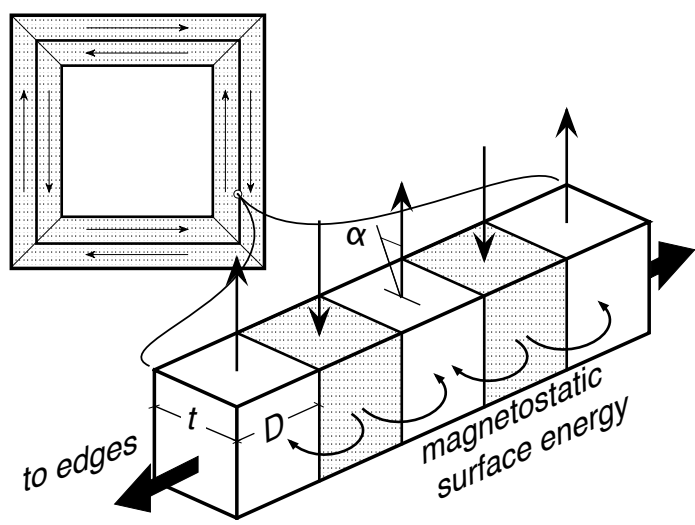

Figure 6. Detail of stripe domains in a demagnetized core. Thin arrows depict magnetic flux - the larger, vertical arrows indicating the dominant flux internal to the core, and the looped arrows depicting the smaller but significant flux crossing the free surface.

to the longer arc length of the domain wall across the foil thickness.

Increasing the applied field further expands the domain wall (Fig. 8a). Eventually a field value is reached where adjacent domain walls make contact (Fig. 8b), at which point a domain wall reconnection occurs (Fig. 8c), forming a new configuration in which a pair of arched domains

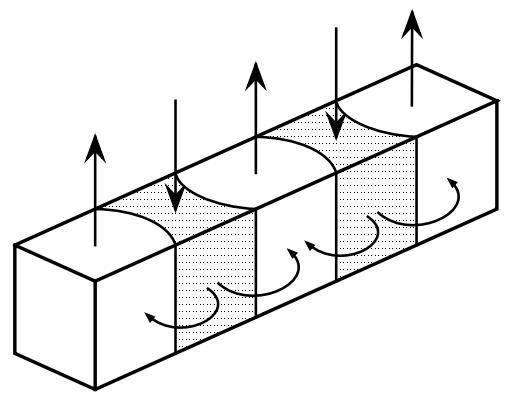

Figure 7. Domain wall motion for initial permeability.

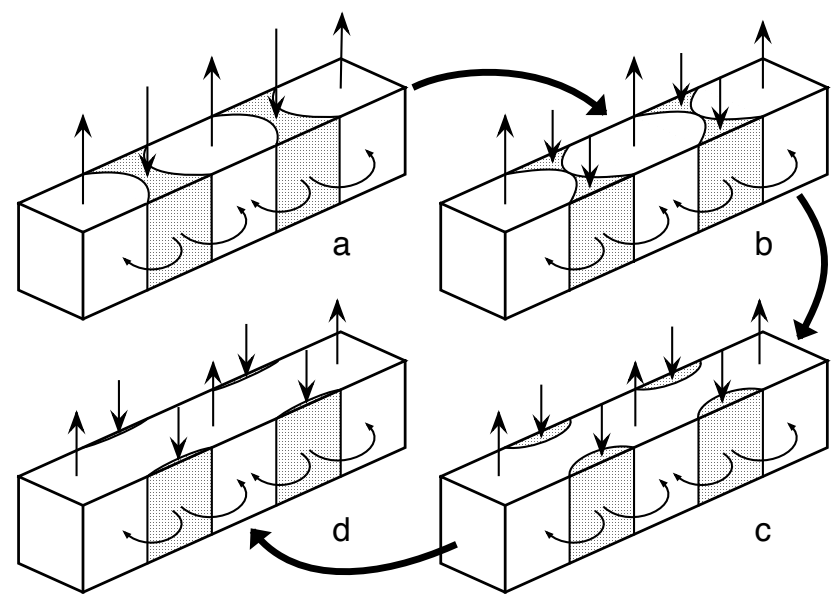

Figure 8. Stripe and channel domain configurations.

is left to satisfy the magnetostatic energy boundary condition. The domain wall area is reduced by this reconnection. The event is irreversible and exothermic, with excess energy dissipated thermally as eddy currents. Such an irreversible event must have random activation energy and thus must be a source of noise. One can identify such a reconnection with a Barkhausen jump. Further field increases serve to reversibly compress these new domains (Fig. 8d), which I shall call "channel domains" to distinguish them from the original 


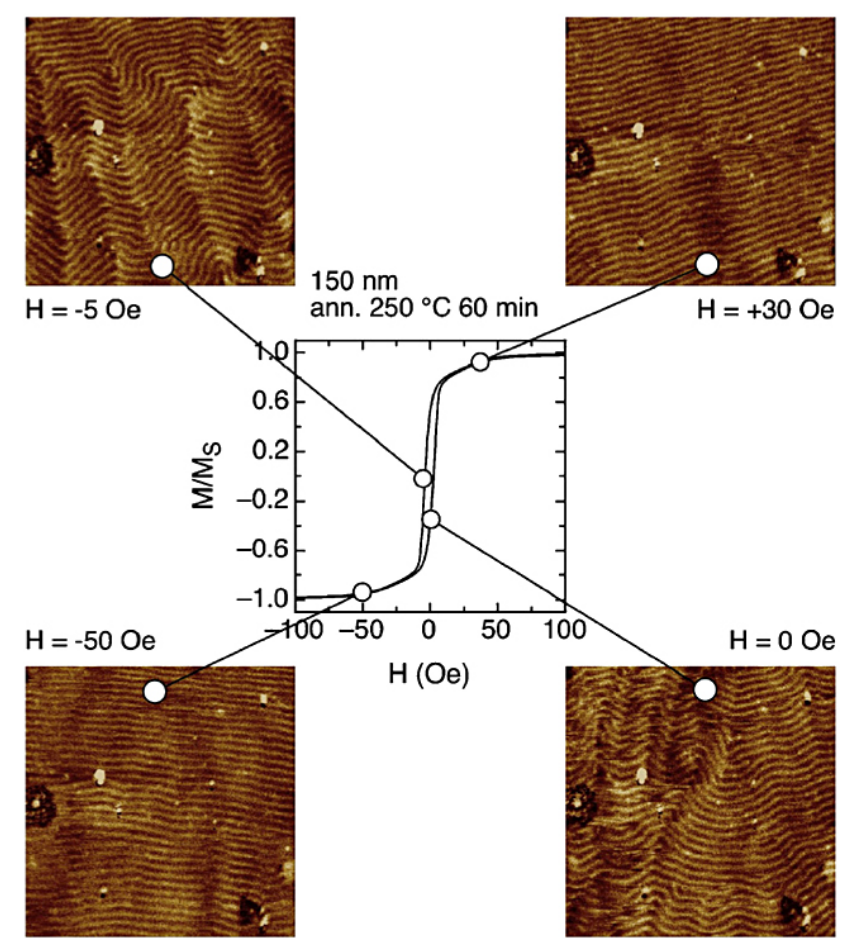

Figure 9. Hysteresis loop of the $150 \mathrm{~nm}$ thick film annealed at $250{ }^{\circ} \mathrm{C}$ for $60 \mathrm{~min}$. Selected MFM images are shown, which are taken at different applied field values. The corresponding point of the hysteresis loop is indicated (Coïsson et al., 2009). (Non-SI units are in the original publication: $1 \mathrm{~A} \mathrm{~m}^{-1} \cong 0.0126 \mathrm{Oe}$.) The specimen was a sputtered $\mathrm{FeSiB}$ amorphous film. Reproduced with permission of the copyright owner.

stripe domains. However the free-surface expression of the channel domains remains, and the domains themselves cannot denucleate.

In Coïsson et al. (2009), Fig. 4 (here as Fig. 9) provides evidence for the formation of channel domains. MFM images of the domains' surface expressions for the two polarizations of a saturated specimen are practically indistinguishable, and substantiate the strength of the magnetostatic energy boundary condition. (To be clear, in crystalline alloys, the stripe domains would form due to magnetocrystalline anisotropy and not spin reorientation (Coïsson et al., 2009; Sharma et al., 2006). Their test material was an ironmetalloid-sputtered film, but arguably the magnetostatic energy constraints should be similar.)

The saturated state is thus one of the channel domains, which, being shallow and bounded by a free surface, are relatively difficult to compress (low permeability). The demagnetized state is dominated by stripe domains. Walls are relatively easy to move, and reconnections manifest as large values of differential permeability (Barkhausen jumps). In general the distribution of individual crystals' easy axes will be random. This results in a range of $H$ values for which reconnections will occur in a bulk specimen, and any attempt

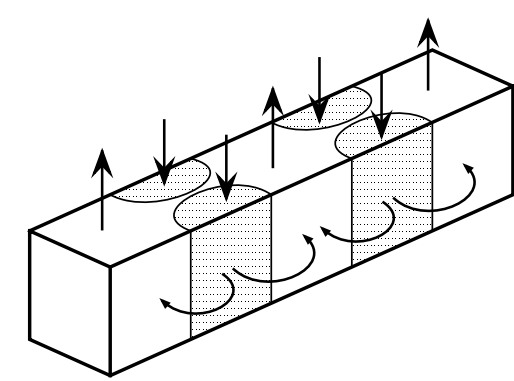

Figure 10. Expanding channel domains.

to estimate differential permeability will need to take this into account. The return from saturation starts by expanding the channel domains, similar to inflating a bubble (Fig. 10). Channel domains self-align across the thickness of the foil, due to the transverse magnetic moments of the channels having opposing magnetic charges, attracting each other by means of a mechanism broadly similar to one found by O'Brien et al. (2009) for transverse domains in permalloy nanowires. Initially the domain walls extend slowly, requiring a lot of variation in $H$ for relatively little gain in net $B$, hence the slowly changing but accelerating tops of the hysteresis curve. The channel domains begin to expand, adding domain wall area and requiring energy to do that - the energy of hysteresis. Eventually the channels themselves reconnect via a second, smaller Barkhausen jump to again form stripe domains, and then the process repeats, now in the opposing polarity. For stripe domains reconnecting into channels, a lot of domain wall energy is released. For channel domains converting to stripes, the amount of energy available is much less clear. The field value at which this occurs is likely closely related to what we can observe as coercivity $H_{\mathrm{c}}$.

Using Eqs. (6) and (7), solving for and eliminating $D$, one finds that for a demagnetized stripe domain system, domain wall energy per unit volume is proportional to

$E_{\mathrm{d}} \propto t^{-1 / 2} \times \alpha \times B_{\mathrm{s}}^{3 / 2} \times K^{1 / 4}$.

A hypothesis that fluxgate noise power is proportional to $E_{\mathrm{d}}$ can now predict all of the principal observations. Noise power increases as foil thickness decreases, or as $B_{\mathrm{S}}$ or anisotropy increase. Equation (8) also predicts that the easy axis dip angle is an important element in creating noise, with small dip angles desired, and may explain some of the results of Fig. 2, whereby lower dip angles lead to reduced magnetostatic energy. Stripe domain reconnection energy should not vary with the rate $\mathrm{d} H_{\mathrm{d}} / \mathrm{d} t$. Therefore energy released per cycle, and thus noise energy per cycle, is independent of a fluxgate's drive frequency, consistent with this author's experience. 


\section{Putting it to the test}

Two of the four parameters given in Eq. (8), $B_{\mathrm{s}}$ and anisotropy, are well known to be involved in producing fluxgate noise. With Marc Lessard and others at University of New Hampshire, I have put foil thickness as a controlling parameter to the test by making 6-81 ring cores that differ from the Infinetics design only in foil thickness. We produced two groups of rings, one with one layer and the other with seven layers, as follows: cast an ingot of $6.0 \% \mathrm{Mo}$, $81.3 \% \mathrm{Ni}, \mathrm{Fe}$ balance. Fabricate flat strips approximately $3 \mathrm{~mm} \times 25 \mathrm{~mm} \times 125 \mathrm{~mm}$. Homogenize at $1100^{\circ} \mathrm{C}$ in $95 \%$ $\mathrm{Ar} / 5 \% \mathrm{H}$ for 7 days. Cold-roll to $100 \mu \mathrm{m}$, with no in-process anneal. Slit to width. Coat with a thin layer of $\mathrm{MgO}$. Spotweld to an Inconel X750 bobbin. Heat treat in $95 \% \mathrm{Ar} / 5 \% \mathrm{H}$ for $4 \mathrm{~h}$ at $1100^{\circ} \mathrm{C}$, cool to $600{ }^{\circ} \mathrm{C}$, and then cool at $35^{\circ} \mathrm{Ch}^{-1}$ to room temperature. At no point during this development was any attempt made to optimize a process step. This was simply our first attempt to fabricate a $6-81$ polycrystalline permalloy ring core.

Noise PSD at $1 \mathrm{~Hz}$ for the two types of $100 \mu \mathrm{m}$ rings are 14 and $5.2 \mathrm{pTrHz}^{-1}$, respectively, the difference consistent with root-mean-square stacking. Figure 11 presents the $B-H$ curves for these and Infinetics 3 and $12 \mu \mathrm{m}$ rings, measured at $25 \mathrm{~Hz}$. All four rings were assembled with $25.4 \mathrm{~mm}$ diameter bobbins. $B-H$ curves were collected using primary and secondary windings of 10 turns each. Each core and a current sense resistor for $H$ were driven by a triangular current source peaking at $\pm 110 \mathrm{~A} \mathrm{~m}^{-1}$. For $B$, secondary signals passed to an operational amplifier configured as a high-input-impedance integrator.

The magnetic material was 6-81 in all cases, and heat treatments were nominally identical. The $100 \mu \mathrm{m}$ ring has a single layer and the $400 \mu \mathrm{m}$ ring has a single circular wire of diameter $400 \mu \mathrm{m}$. The magnetic masses of the four rings match within $20 \%$, allowing the $B-H$ curves to be recorded without any changes to the measurement settings. Between measurements, only the ring was changed. Coercivities for the four rings are in the range 3-7 $\mathrm{A} \mathrm{m}^{-1}$.

\subsection{Another look at $B-H$ loop curvature}

Infinetics thin foils develop high differential permeability and more square $B-H$ curves. The $100 \mu \mathrm{m}$ rings exhibit much "rounder" curves and lower remanence, yet have excellent noise performance. In fact, the new seven-layer rings at $5.2 \mathrm{pT}$ are amongst the best performing permalloy ring cores ever produced. How round $B-H$ curves improve noise performance requires a further development of the noise model.

Referring now to Fig. 11c, from point A to point B there is little curvature, so little or no power is transferred to the sensor winding. Changes in $B$ are dominated by large Barkhausen jumps which are identified in the noise model with domain wall reconnections from stripes to channels. All the energy released by these events couples back into the

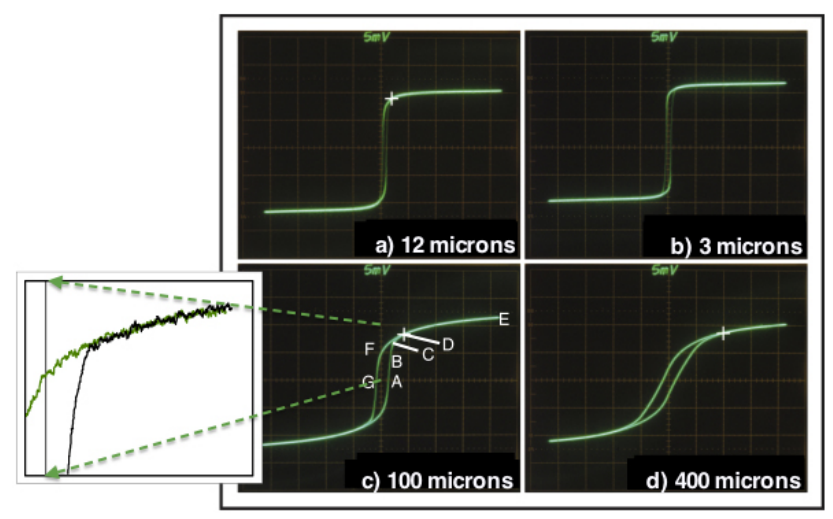

Figure 11. Top panels: Infinetics 12 and $3 \mu \mathrm{m} B-H$ curves. Bottom panels: $6-81100$ and $400 \mu \mathrm{m}$. The drive field is $\pm 110 \mathrm{~A} \mathrm{~m}^{-1}$, a triangle waveform at $25 \mathrm{~Hz}$ to approximate DC behavior. The inset for (c) presents a digitally acquired detail of the distinct transition from irreversible behavior to reversible behavior. The argument presented here is that there are no Barkhausen-jump-related losses above the transition point, and that the large variation in $B$ above the transition point requires reversible domain wall motion in the form of channel domain compression.

$H_{\mathrm{d}}$ drive circuit and no noise energy gets into the sensor winding.

From $\mathrm{B}$ to $\mathrm{D}$, curvature is larger, reaching a maximum at point C. Over this interval, energy is transferred to the sensor winding. Since changes in $B$ are still dominated by stripe-tochannel reconnections in this range, it is here that fluxgate noise power is generated, ending at D, just beyond closure of the hysteresis loop. From $\mathrm{D}$ to $\mathrm{E}$, changes in $B$ can result from domain wall movement in the form of channel domain compression. Our new ring cores exhibit significant curvature in this range, resulting in its round $B-H$ curve. This curvature generates additional sensor energy, and since the process of channel domain compression is nominally a reversible process, this additional sensor energy comes with little noise energy when compared with interval B-D.

The close-up for Fig. 11c is a detailed view showing a well-defined transition in the $100 \mu \mathrm{m}$ data from irreversible behavior to reversible behavior, a transition not noticeable in the return path. Such a sharp transition requires a magnetization process that can support such transitions and that provides for an entirely reversible process above the transition point. Conventional thinking calls for reversible rotation to explain much of the approach to saturation from $\mathrm{D}$ to $\mathrm{E}$ (Chikazumi, 1997, Fig. 18.26), the realignment en masse of individual electronic spins. Being a body force, such realignment should not depend on geometry, particularly material thickness. Comparing the 12 and $100 \mu \mathrm{m}$ curves in Fig. 1 dispels this possibility. The hysteresis loop closure points marked "+" indicate where reversibility begins. Given that all other variables have been constrained, one would expect the two $B-H$ curves to the right of these points to coincide, 


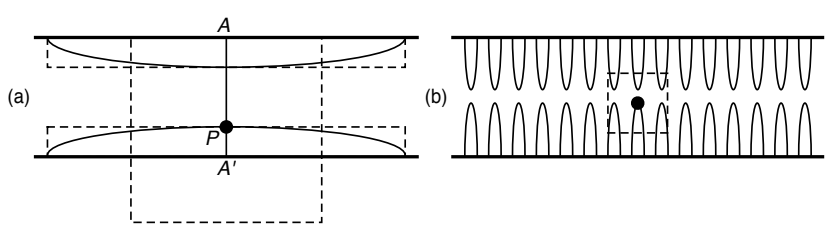

Figure 12. Channel domain sections: (a) thin foils and (b) thick foils.

and clearly this is not the case. Reversible rotation on its own would require an average easy axis misalignment of $45 \%$ to explain the $30 \%$ reversible approach to saturation in Fig. 11c. This is unlikely, implying a need for reversible domain wall motion.

From $\mathrm{E}$ to $\mathrm{D}$ and partway to point $\mathrm{F}$, an interval of channel domain expansion, energy may be recaptured from the sensor winding. From $\mathrm{D}$ to $\mathrm{F}$ the $B-H$ curve is dominated by small Barkhausen jumps which can be identified with channel-tostripe reconnections (Chikazumi, 1997, Fig. 18.43). From point $\mathrm{F}$ to point $\mathrm{G}$ the $B-H$ curve is again of low curvature, dominated by large Barkhausen jumps (stripe-tochannel reconnections).

The channel domain concept can also inform remanence variability in permalloy foils, and in particular why thin foils exhibit greater remanence. Remanence exists because channel domain walls, while satisfying internal boundary conditions, do not lead to a symmetry such that net magnetization is zero.

Figure 12 displays the proposed channel domains for very thin foils (a) (or high anisotropy), or very thick foils (b) (or low anisotropy). For thin foils (Fig. 12a), at point $\mathrm{P}$ on channel domain centerline section $A-A^{\prime}$, the internal boundary condition requires at $\mathrm{P}$ that $H=0$. The very wide aspect of the channel domain means that the field at $\mathrm{P}$ is mainly influenced by a neighborhood dominated by relatively planar domain walls. This neighborhood is depicted as the dashed square. A simple calculation using oval channels leads to a remanence ratio estimate of $59 \%$, in rough agreement with the $B-H$ curves in Fig. 11 .

For thick foils the domains are larger, but their aspects are very different, appearing as thin or dense laminations. A point at the tip of a channel domain is influenced by a much smaller neighborhood. This is depicted in Fig. 12b as the small dashed square. Destructive interference causes material outside this square to have less influence. In the limit, as channels come close to touching, and using ovals to approximate the domain shape, one obtains a remanence ratio of $21 \%$, a value found in many natural materials, and also in this study for the $400 \mu \mathrm{m}$ material. Thicker foils thus reduce fluxgate noise in two ways, both of which reduce total power to the Barkhausen jumps. They reduce total domain wall energy (Pfeifer and Boll, 1969), and deep channels reduce the range in $B$ occupied by the hysteresis loop.
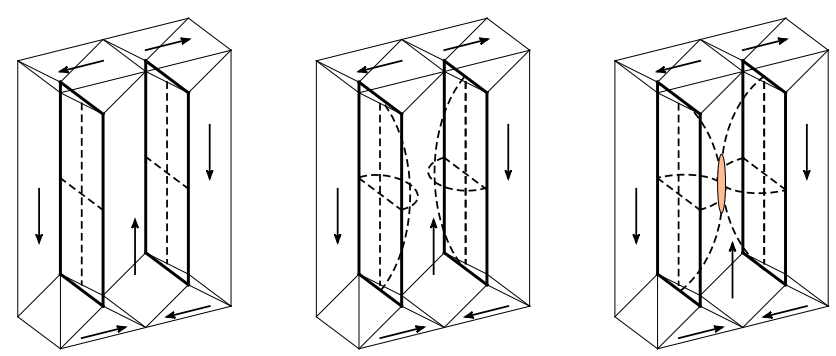

Figure 13. Three-dimensional representation of a single grain and stripe-to-channel reconnection. The left image depicts the unmagnetized stripe domains within a grain. A larger grain would contain additional domains, all of which the same width. The middle image depicts initial permeability. The right image depicts the start of reconnection at the domain wall midpoint. A fixed amount of domain wall displacement is required, regardless of the size of the grain. A larger grain would also have longer stripe domains and require less external field for a given amount of wall displacement, i.e., higher initial permeability. Higher initial permeability in combination with a fixed amount of wall displacement for reconnection implies lower coercivity. Thus increasing grain size leads directly to higher permeability and lower coercivity.

\subsection{Effects of grain size}

In a determination by scanning electron microscopy, grain sizes of our new material are about $20 \mu \mathrm{m}$; thus there exists a large intergrain boundary area within the foil. Mismatch of easy axes at these interior surfaces also serves to localize magnetostatic energy, and if channel domains form at free surfaces, so too should they form at intergrain boundaries. This source of magnetostatic energy must give rise to additional domain walls. Energy needed to reconnect the intergrain channel domains back to stripes will manifest as an increase in hysteresis loss, and relates to the known inverse relation between grain size and coercivity (Couderchon et al., 1989; Herzer, 1990; Pfeifer and Radaloff, 1980). Speculating further, if larger grains lead to longer magnetic domains then considered as a solenoid, each domain's local field near its surface would decrease with increasing grain size. This implies that a given amount of domain wall displacement requires less drive field to achieve equilibrium, and by extension that domain wall reconnections require less external field (Fig. 13). These are interpretable as higher initial permeability and lower coercivity, respectively, both known to correlate with lower fluxgate noise (Nielsen et al., 2010).

Our 6-81 material, with over twice the coercivity of the Infinetics material, must have significantly more magnetostatic energy. A scanning electron micrograph (Kuyucak, 1996) of an Infinetics $3 \mu \mathrm{m}$ ring core showed grains with $10 \mu \mathrm{m}$ average size - much larger than the foil thickness. For the $3 \mu \mathrm{m}$ rings, free-surface magnetostatic energy must dominate its energy budget. 


\section{Additional considerations}

Equation (8) can be used to guide choices in magnetic materials and in ring-core geometry. Regarding the choice of material, 6-81 clearly has very desirable properties. To improve further we need to look for lower $B_{\mathrm{s}}$ materials while simultaneously maintaining low anisotropy. Within the quaternary system $\mathrm{Fe}-\mathrm{Ni}-\mathrm{Mo}-\mathrm{Cu}$, it is possible to estimate $B_{\mathrm{s}}$ by calculating an alloy's average atomic moment: $\mathrm{Bm}=$ at $\% \mathrm{Fe} \cdot 2.8+$ at $\% \mathrm{Ni} \cdot 0.6-$ at $\% \mathrm{Mo} \cdot 4.6-$ at $\% \mathrm{Cu}$ $\cdot 0.4$, and correlating that with $B_{\mathrm{s}}$. Figure 14 summarizes such data. The open square marked " 3 " represents 6-81. The circle marked "6" represents Neumann's "1040" alloy, which is a possible contender for an improved fluxgate core material due to its lower $B_{\mathrm{s}}$.

Reducing magnetostatic energy by reducing free-surface area is an obvious method to reduce domain wall energy. The use of thicker foils or even wires is such a means, with both options being relatively easily achieved. Reducing intergrain surface area is also a means to reduce magnetostatic energy, which is to be achieved by the application of specific heat treatments tailored to growing large crystals. Ideally a typical grain would be the full thickness of the foil or wire and its diameter much larger. Cold forming to control for easy axis direction might also be an approach to further reduce domain wall energy.

\section{Conclusions}

I have presented evidence by means of both data and theory that noise power in the parallel-gated fluxgate has its origin in domain wall energy that transforms irreversibly and exothermically to eddy current energy when domain walls reconnect, identifiable with Barkhausen jumps. Domain wall energy available for noise generation is maintained in a balance with magnetostatic energy, which, in thin foils, is dominated by the anisotropy-driven surface magnetostatic energies. Reducing surface area thus reduces noise energy.

$E_{\mathrm{d}}$ and thus fluxgate noise is reduced by treating the permalloy for low anisotropy. However the biggest influence on $E_{\mathrm{d}}$ is the saturation total magnetic flux density $B_{\mathrm{s}}$. Reducing $B_{\mathrm{S}}$ simultaneously reduces all energies. Its only limitation is a need to concurrently reduce Curie temperature.

These argue for the existence of a previously unidentified magnetic domain state that I have called "channel domains". A channel domain hypothesis can be expressed as follows: in the presence of no external field, an individual crystal of a soft magnetic metal can assume one of three magnetized states. One state, in the form of stripe domains, has zero net magnetization. The second and third states, in the form of channel domains of different polarizations, have nonzero net magnetization. A stripe domain or a channel domain state can convert to the other by domain wall expansion and irreversible reconnection - a large and small Barkhausen jump,

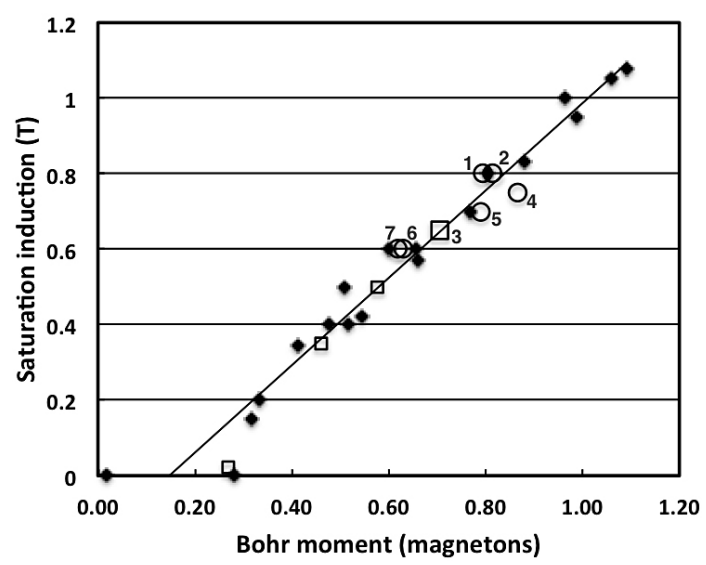

Figure 14. Saturation total magnetic flux density vs. average atomic moment. Data are from Neumann (1934), von Auwers and Neumann (1935) or Bozorth (1993), or are calculated estimates. Large, open symbols represent alloys: (1) $14 \% \mathrm{Cu}$, (2) mumetal, (3) 6-81, (4) 4-79, (5) supermalloy, (6) "1040", and (7) $28 \% \mathrm{Cu}$. Small symbols represent $B_{\mathrm{S}}$ data for a range of ternary alloys.

respectively. The three states, one in the form of stripe domains and the other two in the form of channel domains, can satisfy nearly identical surface boundary conditions. The combination of crystals in a stripe domain state and those in a channel domain state is arbitrary and will depend on the history of external fields. DC hysteresis results from the cycling between stripe and channel domain states via Barkhausen jumps.

Much work remains. Specimens need to be created which have much larger grain sizes than in existing materials. This will require heat treatments at higher temperatures and for longer durations than normally used for permalloys. Domain imaging of large-grain, low-anisotropy crystalline permalloys throughout a magnetization cycle should provide further testing of the hypothesis. Combining domain imaging with the manipulation of final heat treatments could determine the relations between anisotropy, domain sizes and fluxgate noise. Fabric analysis could provide data regarding easy axes alignments. Micromagnetic numerical simulations of stripe domain systems with pinned free-surface expressions could lead to understanding of the energetics of domain wall reconnections.

In the future, careful examinations of reconnection processes might be able to draw connections between micromagnetic mechanisms and large-scale, phenomenological models (Bertotti, 2008). The existence of channel domain states may go a long way in the understanding of many phenomena in magnetically soft polycrystalline metals, including remanence, DC coercivity, and asymmetry in the $B-H$ loop; solving the "domain nucleation problem"; and an irreversible, exothermic process that produces both fluxgate and Barkhausen noise. 
Acknowledgements. I thank J. R. Bennest, G. Cross, and S. Allegretto, who assisted with this manuscript. The Geological Survey of Canada made the collection of 6-81 ring cores available. I thank S. Kuyucak of CANMET; P. Dosanjh at UBC; M. Lessard and the others at UNH; and L. Jewitt, Laura K Jewitt Design Gallery, for their efforts in creating the new 6-81 ring cores.

Edited by: M. Díaz-Michelena

\section{References}

Amos, N. R., Fernandez, R., Ikkawi, R., Lee, B., Lavrenov, A., Krichevsky, A., Litvinov, D., and Khizroev, S.: Magnetic force microscopy study of magnetic stripe domains in sputter deposited Permalloy thin films, J. Appl. Phys., 103, 07E732-107E732-3, doi:10.1063/1.2835441, 2008.

Bertotti, G.: Connection between microstructure and magnetic properties of soft magnetic materials, J. Magn. Magn. Mater., 320, 2436-2442, 2008.

Bozorth, R. M.: Ferromagnetism, in facsimile, edited by: Perkins, W., IEEE Press, Piscataway, NJ, 1993.

Butta, M. and Ripka, P.: Two-Domain Model for Orthogonal Fluxgate, IEEE T. Mag., 44, 3992-3995, 2008.

Chikazumi, S.: Physics of Ferromagnetism, 2nd Edn., Oxford, Clarendon, 1997.

Coïsson, M., Vinai, F., Tiberto, P., and Celegato, F.: Magnetic properties of FeSiB thin films displaying stripe domains, J. Magn. Magn. Mater., 321, 806-809, 2009.

Couderchon, G., Porteseil, J. L., Bertotti, G., Fiorillo, F., and Soardo, G. P.: Magnetization Process in NiFe Alloys with Vanishing Anisotropies, IEEE T. Mag., 25, 3973-3975, 1989.

Gordon, D. I., Lundsten, R. H., Chiarodo, R. A., and Helms Jr., H. H.: A Fluxgate Sensor of High Stability for Low Field Magnetometry, IEEE T. Mag., 4, 397-401, 1968.

Herzer, G.: Grain size dependence of coercivity and permeability in nanocrystalline ferromagnets, IEEE T. Mag., 26, 1397-1402, 1990.

Kittel, C.: Physical Theory of Ferromagnetic Domains, Rev. Modern Phys., 21, 541-583, 1949.

Kuyucak, S.: Characterization of Permalloy Strip, Metals Technology Laboratories Report MTL 96-28, Natural Resources Canada, Ottawa, 1996.

Marshall, S. V.: Sensitivity of the Ring-Core Magnetometer, IEEE T. Mag., 2, 773, 1966.
Narod, B. B., Bennest, J. R., Strom-Olsen, J. O., Nezil, F., and Dunlap, R. A.: An evaluation of the noise performance of $\mathrm{Fe}, \mathrm{Co}$, $\mathrm{Si}$, and $\mathrm{B}$ amorphous alloys in ring-core fluxgate magnetometers, Can. J. Phys., 63, 1468-1472, 1985.

Neumann, H.: Neue magnetische Legierung "1040" mit hoher Anfangs-Permeabilität, Archiv Techn. Messen, Leiniz Verlag, München, 913-915, 1934.

Nielsen, O. V., Afanassiev, Y., and Fornaçon, K. H.: Magnetic Materials for Sensors, in: Fluxgate Magnetometers for Space Research, edited by: Musmann, G., Books on Demand, Nordersted, 152-168, 2010.

O’Brien, L., Petit, D., Zeng, H. T., Lewis, E. R., Sampaio, J., Jausovec, A. V., Read, D. E., and Cowburn, R. P.: Near-Field Interaction between Domain Walls in Adjacent Permalloy Nanowires, Phys. Rev. Lett., 103, 077206, doi:10.1103/PhysRevLett.103.077206, 2009.

Pfeifer, F.: Zum Verständnis der magnetischen Eigenschaften technischer Permalloylegierungen, Z. Metallkd., 57, 295-300, 1966.

Pfeifer, F. and Boll, R.: New Soft Magnetic Alloys for Applications in Modern Electrotechnics and Electronics, IEEE T. Mag., 5, 365-370, 1969.

Pfeifer, F. and Radaloff, C.: Soft magnetic Ni-Fe and Co-Fe alloys some physical and metallurgical aspects, J. Magn. Magn. Mater., 19, 190-207, 1980.

Primdahl, F.: The Fluxgate Mechanism, Part I: The Gating Curves of Parallel and Orthogonal Fluxgates, IEEE T. Mag., 6, 376-383, 1970.

Primdahl, F., Brauer, P., Merayo, J. M. G., and Nielsen, O. V.: The fluxgate ring-core internal field, Measurement Sci. Technol., 13, 1248-1258, 2002.

Sharma, P., Kimura, H., Inoue, A., Arenholz, E., and Guo, J.-H.: Temperature and thickness driven spin-reorientation transition in amorphous Co-Fe-Ta-B thin films, Phys. Rev. B, 73, 052401-1052401-4, doi:10.1103/PhysRevB.73.052401, 2006.

Shirae, K.: Noise in amorphous magnetic materials, IEEE T. Mag., 20, 1299-1301, 1984.

Shishido, H., Kann, T., and Ito, Y.: The Magnetic Domain and Properties of Amorphous Ribbons, IEEE T. Mag., 21, 49-52, 1985.

von Auwers, O. and Neumann, H.: Über Eisen-Nickel-KupferLegierungen hoher Anfangspermeabilität, Wissenschaftliche Veröffentlichungen aus den Siemens-Werken, 14, 92-108, 1935.

Weiss, P. R.: The Application of the Bethe-Peierls Method to Ferromagnetism, Phys. Rev., 74, 1493-1504, 1948. 